\title{
Regulation of drug sensitivity of gastric cancer cells by human calcyclin-binding protein (CacyBP)
}

\author{
Yongquan Shi*, Wenhua Hu*, Fang Yin, Li Sun, Changuiang Liu, Mei Lan, and Daiming Fan \\ Institute of Digestive Diseases, Xijing Hospital, Fourth Military Medical University, Xi'an 710032, Shaanxi Province, China
}

\begin{abstract}
Background. Calcyclin-binding protein (CacyBP) was previously identified as an upregulated gene in a multidrugresistant gastric cancer cell line, SGC7901/ADR, compared to its parental cells, SGC7901, by subtractive hybridization. The aim of this study was to explore the role of CacyBP in multidrug resistance (MDR) in gastric cancer cells.

Methods. The cDNA encoding CacyBP was generated by reverse-transcription-polymerase chain reaction (RT-PCR), and mouse antisera against CacyBP was raised using recombinant CacyBP as the immunogen. The expression of CacyBP in gastric cancer cells was determined by Northern and Western blots. Sense and antisense vectors for CacyBP were introduced into SGC7901 and SGC7901/ADR cells, respectively. The 3-(4,5-dimethylthiazol-2-yl)-2,5-diphenyltetrazolium bromide (MTT) assay was performed to evaluate the drug sensitivity of gastric cancer cells. Flow cytometry was employed to determine adriamycin accumulation and retention in gastric cancer cells.

Results. Northern and Western blots demonstrated upregulation of CacyBP in SGC7901/ADR cells compared to SGC7901 cells. SGC7901-CacyBP and SGC7901/ADR-anCacyBP cells were prepared, in which CacyBP was genetically increased and decreased, respectively. As compared with SGC7901, SGC7901-CacyBP cells exhibited significantly increased $(P<0.01) I_{50}$ values for vincristine, adriamycin, and 5fluorouracil. Meanwhile, as compared with SGC7901/ADR, SGC7901/ADR-anCacyBP cells exhibited significantly decreased $(P<0.01) \mathrm{IC}_{50}$ values for these three drugs. SGC7901CacyBP and SGC7901/ADR-anCacyBP cells displayed no obvious difference $(P>0.05)$ in intracellular adriamycin content compared to their corresponding parental cells.

Conclusions. Upregulation of CacyBP is associated with $M D R$ in gastric cancer cells. CacyBP could regulate the responses of gastric cancer cells to chemotherapy. But the underlying mechanisms of CacyBP-related MDR need further identification.
\end{abstract}

Offprint requests to: D. Fan

Received: January 5, 2004 / Accepted: April 20, 2004

* These two authors contributed equally to this work.
Key words Calcyclin-binding protein (CacyBP) - Neoplasm · Stomach $\cdot$ Drug resistance $\cdot$ Multiple

\section{Introduction}

Calcyclin (S100A6)-binding protein (CacyBP) is a $30-\mathrm{kDa}$ protein that can bind to S100A6 in a $\mathrm{Ca}^{2+}$ dependent manner, which was initially identified in Ehrlich ascites tumor (EAT) cells [1,2]. Northern and Western blots have shown that CacyBP is highly expressed in the mouse and rat brain, liver, spleen, stomach, and in EAT cells, and weakly expressed in the rat lung and kidney [3]. It has been found that the level of CacyBP is increased upon erythropoietin receptor activation and that this increase is accompanied by an increase in c-myc and dpp-1 transcriptional activity via the JAK2 pathway [4,5]. These findings indicate that CacyBP may be involved in a signaling pathway activated by erythropoietin in erythroid cells and in neuronal cells in which erythropoietin receptor is highly expressed. SIP (siah-1 interacting protein), which shows 93\% amino-acid sequence identity with mouse CacyBP, has been characterized as a component of the $\beta$-catenin degradation pathway [6]. This raises the possibility that CacyBP may function via $\mathrm{Ca}^{2+}$-dependent interactions in the protein degradation process. Recently, the $\mathrm{Ca}^{2+}$ dependent translocation of CacyBP in neurons and neuroblastoma NB-2a cells has been described [7]. In addition, translocation and phosphorylation of CacyBP during retinoic acid-induced neuronal differentiation of neuroblastoma SH-SY5Y cells has also been reported [8]. However, the physiological and pathological roles of CacyBP still remain unclear.

By a modified subtractive hybridization method, we previously characterized the differentially expressed gene profiles between a multidrug-resistant human gastric cancer cell line SGC7901/ADR and its parental cell 
line SGC7901 [9]. CacyBP (AF314752) was thus found to be upregulated in SGC7901/ADR cells, which indicated an association of this gene with multidrug resistance (MDR). The aim of this study was to explore the role of CacyBP in MDR in gastric cancer cells.

\section{Materials and methods}

\section{Cell culture}

The human gastric adenocarcinoma cell line SGC7901 and its multidrug-resistant variant SGC7901/ADR were cultivated in RPMI 1640 (GIBCO, Grand Island, NY, USA) supplemented with $10 \%$ heat-inactivated fetal calf serum, penicillin $(100 \mathrm{U} / \mathrm{ml})$ and streptomycin $(100 \mu \mathrm{g} / \mathrm{ml})$, in a $\mathrm{CO}_{2}$ incubator (Forma Scientific, Marjetta, OH, USA). SGC7901/ADR cells were maintained continuously in the presence of $0.6 \mu \mathrm{g} / \mathrm{ml}$ adriamycin. The drug was removed from the medium 7 days prior to use.

\section{$R N A$ extraction and reverse-transcription polymerase chain reaction ( $R T-P C R)$}

Total RNA was isolated and purified from gastric cancer cells with a UNIQ-10 total RNA minipreps super kit, according to the protocol given by the manufacturer (Sangon, Shanghai, China). The cDNA encoding CacyBP was generated by RT-PCR, which was performed according to standard protocol. The specific primers for CacyBP were 5'-ACCATGGCTTCAGAA GAGC-3' (sense) and 5'-TCACAGTTCCCAAAACG AC-3' (antisense). PCR products were cloned into pUCm-T vector (Sangon) by a thymine-adenine (T-A) cloning technique. The cDNA inserts were identified by DNA sequencing.

\section{Northern blot analysis}

Fifteen micrograms of total RNA was subjected to formaldehyde-agarose gels electrophoresis, transferred to a nitrocellulose membrane by pressure blotting, and fixed by ultraviolet light crosslinking according to standard protocols. The PCR-generated cDNA was used as a specific probe for CacyBP. Then $\left[\alpha-{ }^{32} \mathrm{P}\right] \mathrm{dCTP}-$ labeled cDNA probes were generated using the PrimeA-Gene-Labeling system (Promega, Madison, WI, USA). Prehybridization was performed overnight at $42^{\circ} \mathrm{C}$ in $5 \times$ standard saline citrate (SSC), $50 \%$ formamide, $5 \times$ Denhardt's reagent, $0.2 \%$ sodium dodecyl sulfate (SDS), and $0.1 \mathrm{mg} / \mathrm{ml}$ sheared salmon sperm DNA. Radiolabeled cDNA probes were hybridized overnight at $42^{\circ} \mathrm{C}$ in a solution identical to the prehybridization solution. Membranes were washed twice with $2 \times \mathrm{SSC} / 0.1 \% \mathrm{SDS}$ at room temperature, twice with $0.2 \times \mathrm{SSC} / 0.1 \% \mathrm{SDS}$ at room temperature, and twice with $0.2 \times \mathrm{SSC} / 0.1 \% \mathrm{SDS}$ at $56^{\circ} \mathrm{C}$. The membrane was then exposed to X-ray film in a dark cassette for $24 \mathrm{~h}$.

\section{Preparation of antisera}

The cDNA inserts for CacyBP were subcloned from recombinant $\mathrm{pUCm}-\mathrm{T}$ vector into $\mathrm{pQE30}$ vector (Qiagen, Hilden, Germany), in which the target gene was fused with histidine tag $(6 \times$ His $)$. The recombinant fusion proteins were expressed in Escherichia coli strain M15 (Qiagen) and used as the immunogen to raise mouse antisera, according to D'Souza et al. [10], with some modification. In brief, recombinant proteins were separated by SDS polyacrylamide gel electrophoresis (PAGE). After staining with $0.25 \mathrm{~mol} / \mathrm{l}$ potassium chloride, the portion of the polyacrylamide gel corresponding to recombinant proteins was cut out and crushed in phosphate-buffered saline (PBS). BALB/c mice were injected subcutaneously with gel particles containing about $10 \mu \mathrm{g}$ recombinant proteins emulsified with complete Freund's adjuvant (Sigma, St. Louis, MO, USA). Three booster injections with gel particles emulsified with incomplete Freund's adjuvant (Sigma) were given at 2-week intervals. Animals were bled 1 week after the last immunization and sera were characterized by Western blot.

\section{Plasmid construction and gene transfection}

To prepare the sense vector pcDNA3.1-CacyBP and the antisense vector pcDNA3.1-anCacyBP, the cDNA inserts for CacyBP were released from recombinant pUCm-T plasmids by restriction enzyme digestion and then cloned into eukaryotic expression vector pcDNA3.1 (Invitrogen, Carlsbad, CA, USA) in forward and backward orientation, respectively. By mediation of Lipofectamine 2000 (GIBCO), the sense and antisense vectors were introduced into SGC7901 and SGC7901/ADR cells, respectively. Forty-eight hours later, cells were placed in growth medium containing G418 (GIBCO) for clone selection. The expression levels of CacyBP in G418-resistant clones were evaluated by Western blot analysis. Finally, two transfectants were obtained, and designated as SGC7901-CacyBP and SGC7901/ADR-anCacyBP, respectively. Meanwhile, the backbone plasmid pcDNA3.1 was introduced into SGC7901 and into SGC7901/ADR cells. The resulting cell sublines were designated as SGC7901pcDNA and SGC7901/ADR-pcDNA, and used as negative controls in subsequent assays. 


\section{Western blot analysis}

Gastric cancer cells in logarithmic phase were harvested, and total cellular proteins were prepared with lysis buffer ( $\mathrm{pH}$ 8.0) containing 1\% NP-40 (Sangon), $50 \mathrm{mM}$ Tris- $\mathrm{HCl}, 150 \mathrm{mM} \mathrm{NaCl}, 0.1 \mathrm{mM}$ phenylmethylsulfonyl fluoride and $1 \mu \mathrm{g} / \mathrm{ml}$ Aprotinin (Sigma). The detergent-soluble fractions were subjected to SDS-PAGE according to standard protocol. Separated proteins were electrotransferred to a nitrocellulose filter. Membranes were blocked with $5 \%$ fat-free milk powder at room temperature for $2 \mathrm{~h}$ and incubated overnight with primary antibodies at $4{ }^{\circ} \mathrm{C}$. Signal detection was performed using an ECL + Plus kit (Amersham Pharmacia, Uppsala, Sweden), according to the manufacturer's instructions. Primary antibodies included the mouse antisera against CacyBP, MGr1 [11], mouse anti-S100A1 (Sigma), mouse anti-S100A6 (Sigma), rabbit anti-S100B (Research Diagnostics, Flanders, NJ, USA), mouse anti-human S100P (Research Diagnostics), and mouse IgG against beta-actin (Boster Biotechnology, Wuhan, Hubei, China). Horseradish peroxidase-conjugated goat anti-mouse $\mathrm{IgG}$ and goat anti-rabbit IgG were obtained from Boster Biotechnology.

\section{In vitro drug sensitivity assay}

Vincristine, adriamycin, cisplatin, and 5-fluorouracil were all freshly prepared before each experiment. Drug sensitivity was evaluated using the 3-(4,5dimethylthiazol-2-yl)-2,5-diphenyl-tetrazolium bromide (MTT) assay as described previously [12]. Briefly, gastric cancer cells in logarithmic phase were harvested and plated into 96 -well plates $\left(1 \times 10^{4}\right.$ cells/well $)$. After culture overnight at $37^{\circ} \mathrm{C}$, the medium was replaced with fresh growth medium containing various concentrations of drugs. Each treatment group was done in triplicate. After cells had been cultured for $72 \mathrm{~h}, 50 \mu \mathrm{l}$ of $2 \mathrm{mg} / \mathrm{ml}$ MTT (Sigma) was added to each well, and the cells were cultured for another $4 \mathrm{~h}$. Then, supernatants were discarded and $150 \mu \mathrm{l}$ of dimethylsulfoxide (DMSO; Sigma) was added to each well to dissolve crystals. The $\mathrm{A}_{490}$ values were read on a spectrophotometer (MPR-2100; Syntron, Carlsbad, CA, USA). Cell survival rates were calculated according to the formula: survival rate $=\left(\right.$ mean $\mathrm{A}_{490}$ of treated wells/mean $\mathrm{A}_{490}$ of untreated wells $) \times 100 \%$. Finally, dose-effect curves of anticancer drugs were drawn on semilogarithm coordinate paper, and $\mathrm{IC}_{50}$ values were determined.

\section{Fluorescence intensity assay of intracellular adriamycin}

The fluorescence intensity of intracellular adriamycin was determined by flow cytometry, as described previ- ously [11]. Briefly, gastric cancer cells in logarithmic phase were seeded into six-well plates $\left(1 \times 10^{6}\right.$ cells/ well) and cultured overnight at $37^{\circ} \mathrm{C}$. After addition of adriamycin to the final concentration of $5 \mu \mathrm{g} / \mathrm{ml}$, cells continued to be cultured for $1 \mathrm{~h}$. Then, cells were trypsinized and harvested (for detection of adriamycin accumulation), or, alternatively, they were cultured in drug-free RPMI 1640 for another $30 \mathrm{~min}$, followed by trypsinization and harvesting (for detection of adriamycin retention). Cells were washed twice with ice-cold PBS, and the fluorescence intensity of intracellular adriamycin was determined using flow cytometry, with an excitation wavelength of $488 \mathrm{~nm}$ and emission wavelength of $575 \mathrm{~nm}$. Finally, the adriamycin-releasing index of gastric cancer cells was calculated according to the formula: releasing index $=$ (accumulation value retention value)/ accumulation value.

\section{Statistical analysis}

Data values were expressed as means \pm SD. Differences were compared by one-way analysis of variance (ANOVA), follwed by Dunnett's multiple comparison tests. A value of $P<0.05$ was considered significant.

\section{Results}

Detection of CacyBP in gastric cancer cells by Northern blot

Although SGC7901/ADR cells were selected with the single anticancer drug adriamycin, they also displayed cross-resistance to other anticancer drugs, such as vincristine, etoposide, cisplatin, 5-fluorouracil, and mitomycin C. Our previous studies have suggested upregulation of CacyBP in SGC7901/ADR cells compared to SGC7901 cells. In the present study, we further evaluated the expression level of CacyBP mRNA in gastric cancer cells by Northern blot. A CacyBP cDNA fragment generated by RT-PCR was used as a specific probe. A radiolabeled 1.0-kb cDNA fragment, according to glyceraldehyde-3-phosphate dehydrogenase (GAPDH), served as the internal control. As the results indicated (Fig. 1), SGC7901/ADR cells exhibited much higher expression of CacyBP mRNA than SGC7901 cells.

\section{Detection of CacyBP in gastric cancer cells by Western blot}

Using recombinant proteins expressed in Escherichia coli as the immunogen, we successfully prepared mouse antisera against CacyBP. As Fig. 2 suggests, this antisera bound to the recombinant CacyBP and detected a single protein band of about $30 \mathrm{kDa}$ from the total 


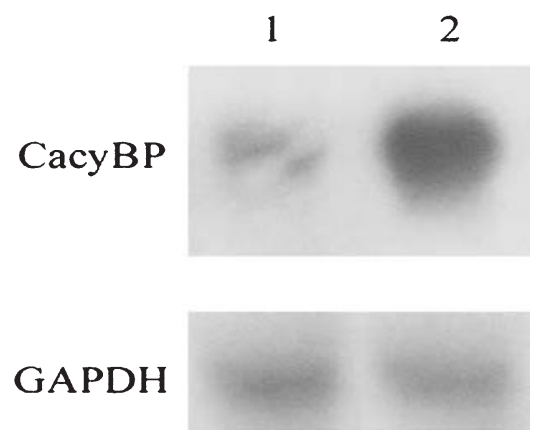

Fig. 1. Detection of calcyclin-binding protein (CacyBP) in gastric cancer cells by Northern blot. Total RNAs extracted from SGC7901 cells (lane 1) and SGC7901/ ADR cells (lane 2) were subjected to Northern blot. The CacyBP cDNA fragment generated by reverse transcriptionpolymerase chain reaction (RT-PCR) was used as a specific probe. A radiolabeled $1.0-\mathrm{kb}$ cDNA fragment, according to glyceraldehyde-3-phosphate dehydrogenase (GAPDH), served as the internal control

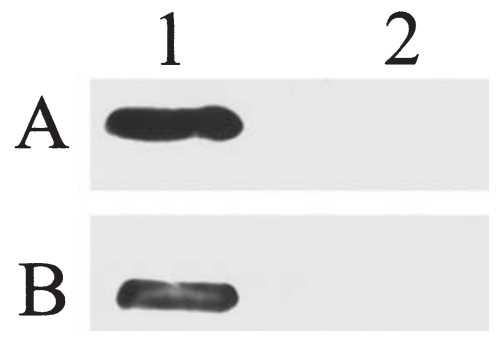

Fig. 2. Characterization of mouse antisera against CacyBP by Western blot. The recombinant CacyBP proteins expressed in Escherichia coli (row $A$ ) and the total cellular proteins extracted from SGC7901/ADR cells (row B) were subjected to Western blot. The antisera against CacyBP were used as the primary antibody (lane 1). The pre-immunized mouse sera were used as control (lane 2). Signal detection was performed using the ECL+ Plus kit (Amersham Pharmacia)

cellular proteins of SGC7901/ADR cells, while preimmunized mouse sera did not react with these two proteins. The antisera were thus used in Western blot to detect CacyBP in gastric cancer cells. As the results showed (Fig. 3), the expression level of CacyBP protein in SGC7901/ADR cells was much higher than that in SGC7901 cells. SGC7901-CacyBP cells, in which the sense vector of CacyBP was introduced, displayed significantly increased expression of CacyBP compared to SGC7901 cells. SGC7901/ADR-anCacyBP cells, in which the antisense vector of CacyBP was introduced, displayed significantly decreased expression of CacyBP compared to SGC7901/ADR cells. SGC7901-pcDNA and SGC7901/ADR-pcDNA cells, in which the backbone plasmid pcDNA3.1 was introduced, showed no obvious changes of CacyBP expression as compared with their corresponding parental cells.

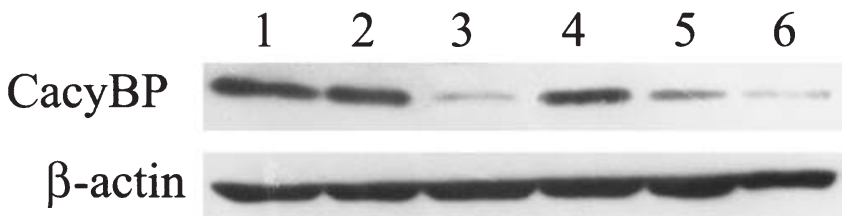

Fig. 3. Detection of CacyBP in gastric cancer cells by Western blot. Six cell lines were evaluated: human gastric cancer cells SGC7901 (lane 6), SGC7901-pcDNA (transfected with the backbone plasmid pcDNA3.1; lane 5), SGC7901-CacyBP (transfected with CacyBP sense vector; lane 4), multidrugresistant gastric cancer cells SGC7901/ADR (lane 1), and SGC7901/ADR-pcDNA (transfected with the backbone plasmid pcDNA3.1; lane 2), and SGC7901/ADR-anCacyBP (transfected with CacyBP antisense vector; lane 3). The mouse antisera against CacyBP were used as the primary antibody. Signal detection was performed using the ECL + Plus kit. $\beta$ Actin was used as the internal control

\section{Regulation of MDR in gastric cancer cells by CacyBP}

SGC7901-CacyBP and SGC7901/ADR-anCacyBP, the two cell lines in which CacyBP was genetically increased or decreased, were used as cell models to study the regulatory effects of CacyBP on the drug sensitivity of gastric cancer cells. As Table 1 shows, as compared with SGC7901 and SGC7901-pcDNA cells, SGC7901CacyBP cells exhibited significantly increased $(P<$ $0.01) \mathrm{IC}_{50}$ values for vincristine, adriamycin, and 5fluorouracil. Meanwhile, as compared with SGC7901/ ADR and SGC7901/ADR-pcDNA cells, SGC7901/ ADR-anCacyBP cells exhibited significantly decreased $(P<0.01) \mathrm{IC}_{50}$ values for vincristine, adriamycin, and 5-fluorouracil. These data suggested that the expression of CacyBP was negatively correlated with the drug sensitivity of gastric cancer cells. However, CacyBP had no obvious effect on the sensitivity of gastric cancer cells to cisplatin.

\section{Effects of CacyBP on intracellular adriamycin accumulation}

Intracellular drug accumulation and retention were evaluated using adriamycin as probe. The fluorescence intensity of intracellular adriamycin was determined by flow cytometry, and the adriamycin-releasing index was calculated. As the results indicated (Table 2), SGC7901/ ADR cells exhibited significantly decreased $(P<0.01)$ adriamycin-accumulation and retention and a significantly increased $(P<0.01)$ adriamycin-releasing index as compared with SGC7901 cells. However, SGC7901CacyBP and SGC7901/ADR-anCacyBP cells showed no significant $(P>0.05)$ differences in adriamycin accumulation and retention or in the adriamycin-releasing index compared to their corresponding controls, which suggested that CacyBP did not affect intracellular drug accumulation in gastric cancer cells. 
Table 1. $\mathrm{IC}_{50}$ values $(\mu \mathrm{g} / \mathrm{ml})$ of gastric cancer cells for anticancer drugs

\begin{tabular}{lllll}
\hline & \multicolumn{1}{c}{ Vincristine } & Adriamycin & 5-Fluorouracil & Cisplatin \\
\hline SGC7901 & $0.44 \pm 0.09$ & $0.15 \pm 0.04$ & $0.60 \pm 0.11$ & $0.42 \pm 0.07$ \\
SGC7901-pcDNA & $0.67 \pm 0.13$ & $0.14 \pm 0.05$ & $0.83 \pm 0.23$ & $0.69 \pm 0.14$ \\
SGC7901-CacyBP & $2.58 \pm 0.49^{*}$ & $2.89 \pm 0.53 *$ & $3.80 \pm 0.86^{*}$ & $0.54 \pm 0.14$ \\
SGC7901/ADR & $4.87 \pm 0.78^{* *}$ & $4.26 \pm 0.68^{* *}$ & $6.75 \pm 0.95^{* *}$ & $1.51 \pm 0.42^{* *}$ \\
SGC7901/ADR-pcDNA & $4.17 \pm 0.66$ & $4.38 \pm 0.65$ & $6.22 \pm 0.87$ & $1.59 \pm 0.44$ \\
SGC7901/ADR-anCacyBP & $1.35 \pm 0.39 * *$ & $0.82 \pm 0.17 * * *$ & $2.46 \pm 0.43^{* * *}$ & $1.47 \pm 0.41$ \\
\hline
\end{tabular}

One-way analysis of variance (ANOVA) followed by Dunnett's multiple comparison tests revealed statistical differences of $* P<0.01$ vs SGC7901 and SGC7901-pcDNA cells; $* * P<0.01$ vs SGC7901 cells; and $* * * P<0.01$ vs SGC7901/ADR and SGC7901/ADR-pcDNA cells Survival rates of gastric cancer cells to anticancer drugs were evaluated by MTT assay, as described in "Materials and methods". The dose-effect curves of anticancer drugs were drawn on semi-logarithm coordinate paper and, thus, $\mathrm{IC}_{50}$ values were determined. Data values are expressed as means $\pm \mathrm{SD}$ of four independent experiments

Table 2. Fluorescence intensity of intracellular adriamycin, and adriamycin-releasing index of gastric cancer cells

\begin{tabular}{lccl}
\hline & Accumulation & Retention & Releasing index \\
\hline SGC7901 & $28.46 \pm 5.82$ & $15.42 \pm 3.58$ & $0.458 \pm 0.039$ \\
SGC7901-pcDNA & $29.17 \pm 6.11$ & $15.93 \pm 3.94$ & $0.454 \pm 0.041$ \\
SGC7901-CacyBP & $27.26 \pm 5.79$ & $15.19 \pm 3.27$ & $0.443 \pm 0.037$ \\
SGC7901/ADR & $8.54 \pm 1.78^{*}$ & $1.53 \pm 0.36^{*}$ & $0.823 \pm 0.063^{*}$ \\
SGC7901/ADR-pcDNA & $8.66 \pm 1.58$ & $1.38 \pm 0.34$ & $0.852 \pm 0.068$ \\
SGC7901/ADR-anCacyBP & $8.99 \pm 1.74$ & $1.34 \pm 0.31$ & $0.851 \pm 0.057$ \\
\hline
\end{tabular}

One-way ANOVA followed by Dunnett's multiple comparison tests suggested significant differences; $* P<0.01$ vs SGC7901 cells

Gastric cancer cells in six-well plates were treated with $5 \mu \mathrm{g} / \mathrm{ml}$ of adriamycin for $1 \mathrm{~h}$. Cells were trypsinized and harvested (for detection of adriamycin accumulation), or alternatively, cultured in drug-free RPMI 1640 for another $30 \mathrm{~min}$ followed by trypsinization and harvesting (for detection of adriamycin retention). The fluorescence intensity of intracellular adriamycin was determined using flow cytometry with an excitation wavelength of $488 \mathrm{~nm}$ and emission wavelength of $575 \mathrm{~nm}$. The adriamycin-releasing index of gastric cancer cells was calculated according to the formula: releasing index $=$ (accumulation value - retention value $) /$ accumulation value. Each experiment was conducted in triplicate. Data values are expressed as means $\pm \mathrm{SD}$

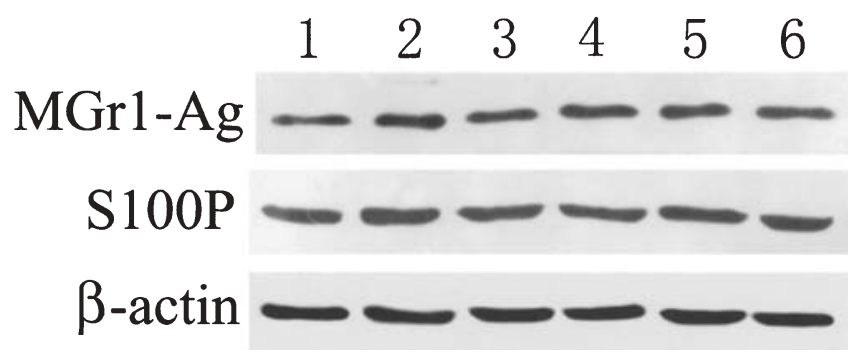

Fig. 4. Detection of MGr1-Ag and S $100 \mathrm{P}$ proteins in gastric cancer cells by Western blot. Six cell lines were evaluated: human gastric cancer cells SGC7901 (lane 1), SGC7901pcDNA (transfected with the backbone plasmid pcDNA3.1; lane 2), SGC7901-CacyBP (transfected with CacyBP sense vector; lane 3), multidrug-resistant gastric cancer cells SGC7901/ADR (lane 6), SGC7901/ADR-pcDNA (transfected with the backbone plasmid pcDNA3.1; lane 5), and SGC7901/ADR-anCacyBP (transfected with CacyBP antisense vector; lane 4). The monoclonal antibody MGr1 and mouse anti-human S 100P were used as primary antibodies. Signal detection was performed using the ECL+ Plus kit. $\beta$-Actin was used as the internal control

\section{Influence of CacyBP on MGrl-Ag and S 100 proteins}

The expression of MGr1-Ag and the expression of several S 100 proteins in gastric cancer cells were determined by Western blot. MGr1-Ag was moderately expressed in SGC7901 cells and slightly increased in SGC7901/ADR cells. However, the transfectants did not show obvious differences in MGr1-Ag expression compared to that in SGC7901 or SGC7901/ADR cells (Fig. 4). S 100A1, S 100A6, and S 100B were not detected in SGC7901 or SGC7901/ADR or their transfectants (data not shown). S 100P was expressed, without significant differences between the six cell lines tested (Fig. 4). These findings suggest that CacyBP has no obvious influence on the expression of MGr1-Ag and S 100P. 


\section{Discussion}

MDR is usually used to describe a resistance phenotype in which cells become simultaneously resistant to different drugs with no obvious structural resemblances and with different cellular targets [13]. MDR is the main cause of chemotherapy failure in malignant tumors, including gastric cancer [14]. Frequently, MDR is intrinsic to the cancer, but as chemotherapy becomes more and more effective, acquired MDR also becomes a common phenomenon. The most common reason for the acquisition of resistance to a broad range of anticancer drugs is the expression of one or more energy-dependent transporters that detect and eject anticancer drugs from cells $[15,16]$. These transporters include P-glycoprotein (P$\mathrm{gp}), \mathrm{MDR}$-associated protein (MRP), lung-resistance protein (LRP), and breast carcinoma-resistance protein. However, it has been confirmed that not all gastric cancer cells or tissues express these transporters [17-20]. Recently, Fan et al. [21], from our institute, examined the expression of glutathione S-transferase (GST)-pi, MRP, LRP, P-gp, and MGr1-Ag in 50 Chinese patients with primary gastric cancer and without any prior chemotherapy. The results revealed that the positive ratios of GST-pi mRNA, MRP mRNA, LRP mRNA, P-gp mRNA, and MGr1-Ag protein in gastric cancer specimens was $36.0 \%, 12.0 \%, 10.0 \%, 10.0 \%$, and $18.0 \%$, respectively, and that, overall, the positive ratio of their expression was only $58.0 \%$. It has been reported that increased expression of thymidine kinase, equilibrative nucleoside transporter 1, and methylenetetrahydrofolate dehydrogenase, as well as decreased expression of orotate phosphoribosyltransferase, thymidine phosphorylase, uridine phosphorylase, cytidine deaminase, and integrin alpha3 were correlated with a 5-fluorouracil-resistant phenotype of gastric cancer cells [22,23]. In another report, overexpression of annexin I and thioredoxin was found to be associated with daunorubicin-resistant, and overexpression of thioredoxin was found to be associated with mitoxantrone-resistant phenotypes of gastric cancer cells [24]. These data strongly suggested there might be diverse regulatory systems in gastric cancer cells under drug attack.

To better understand the regulatory network underlying MDR in gastric cancer cells, we have prepared an adriamycin-resistant gastric cancer cell line, SGC7901/ ADR, which developed resistance not only to adriamycin but also to vincristine, 5-fluorouracil, cisplatin, and mitomycin C [25]. By a modified subtractive hybridization method, we previously found upregulation of CacyBP in SGC7901/ADR cells, which indicated an association of this gene with MDR [9]. In the present study, we confirmed upregulation of CacyBP mRNA in SGC7901/ADR cells by Northern blot, and further the
CacyBP protein was detected in gastric cancer cells by the mouse antisera against CacyBP. The results indicated overexpression of CacyBP in the multidrugresistant gastric cancer cells SGC7901/ADR. However, SGC7901/VCR, another multidrug-resistant cell variant derived from SGC7901, does not show upregulation of CacyBP compared to SGC7901 cells [9]. Similarly, other authors have reported differentially expressed gene profiles in a series of drug-resistant cell variants derived from the same parental cell line [24]. These data also suggest that there might be diverse regulatory systems in tumor cells under drug attack.

To explore the regulatory effects of CacyBP on the multidrug-resistant phenotype of gastric cancer cells, we introduced a sense vector for CacyBP into SGC7901 cells and an antisense vector for CacyBP into SGC7901/ ADR cells. After confirmation of the successful expression of the target gene by Western blot, the MTT assay was used to evaluate the drug sensitivity of the gastric cancer cells. We found that overexpression of CacyBP promoted resistance of the gastric cancer cells SGC7901 to adriamycin, vincristine, and 5-fluorouracil. We also found that downregulation of CacyBP sensitized the multidrug-resistant gastric cancer cells SGC7901/ADR to these three anticancer drugs. These data directly linked CacyBP to MDR in gastric cancer cells, and suggested that CacyBP could be a potential MDR marker and a potential target for MDR reversal therapy in gastric cancer cells. However, it should be noted that CacyBP did not affect the sensitivity of gastric cancer cells to cisplatin, which suggests that CacyBP is just one of the regulatory molecules in SGC7901/ADR cells.

In our previous report [25], decreased drug accumulation mediated by P-gp and MRP, and resistance to druginduced apoptosis, mediated by the upregulation of Bcl-2 and downregulation of Bax, were confirmed to be associated with the multidrug-resistant phenotype of SGC7901/ADR cells. The present study demonstrated significantly decreased adriamycin accumulation and retention, and a significantly increased adriamycinreleasing index in SGC7901/ADR cells compared to SGC7901 cells. The effects of CacyBP on adriamycin accumulation and retention in gastric cancer cells were determined. The results indicated that CacyBP had no obvious influence on adriamycin accumulation and retention. MGr1-Ag, which was initially found by us to be associated with MDR and to affect intracellular adriamycin content in gastric cancer cells [11,12], was also detected in CacyBP-related transfectants. However, no obvious correlation between CacyBP and MGr1-Ag was found in the present study. These data suggested that CacyBP confers MDR in gastric cancer cells, but not through alterations in drug accumulation.

It has been reported that CacyBP bound S 100A6, S 100A1, S 100B, and S 100P, but not S 100A4, calbindin 
$\mathrm{D}$, parvalbumin, or calmodulin [26]. Therefore, we evaluated the expression of S 100A1, S 100A6, S 100B, and $\mathrm{S} 100 \mathrm{P}$ in gastric cancer cells by Western blot. Only S 100P was found to be expressed in SGC7901 and SGC7901/ADR cells. It was recently reported that S $100 \mathrm{P}$ was significantly upregulated in gastric cancer cells treated with retinoic acid [27], which suggested a potential role of $\mathrm{S} 100 \mathrm{P}$ in retinoic acid resistance. In the present study, however, there was no obvious difference in S 100P expression among the six cell lines tested (SGC7901 and SGC7901/ADR and their transfectants). Thus, further exploration is still needed to determine the roles of S 100P and other S 100 proteins in CacyBPrelated MDR in gastric cancer cells.

In summary, the present study adds CacyBP to the players that regulate the responses of gastric cancer cells to chemotherapy. CacyBP could be used as a potential MDR marker and a potential target for MDR reversal therapy in gastric cancer cells. But the underlying mechanisms of CacyBP-related MDR need further identification.

Acknowledgment This work was supported by the National Natural Science Foundation of China (grants No. 30030140 and No. 30024002) and "863" project of China (grant No. 2004AA227080).

\section{References}

1. Filipek A, Wojda U. p30, a novel protein target of mouse calcyclin (S100A6). Biochem J 1996;320:585-7.

2. Filipek A, Kuznicki J. Molecular cloning and expression of a mouse brain cDNA encoding a novel protein target of calcyclin. J Neurochem 1998;70:1793-8.

3. Jastrzebska B, Filipek A, Nowicka D, Kaczmarek L, Kuznicki J. Calcyclin (S100A6) binding protein (CacyBP) is highly expressed in brain neurons. J Histochem Cytochem 2000;48:1195202.

4. Xia ZB, Dai MS, Magoulas C, Broxmeyer HE, Lu L. Differentially expressed genes during in vitro differentiation of murine embryonic stem cells transduced with a human erythropoietin receptor cDNA. J Hematother Stem Cell Res 2000;9:651-8.

5. Pircher TJ, Geiger JN, Zhang D, Miller CP, Gaines $\mathrm{P}$, Wojchowski DM. Integrative signaling by minimal erythropoietin receptor forms and c-Kit. J Biol Chem 2001;276:8995-9002.

6. Matsuzawa SI, Reed JC. Siah-1, SIP, and Ebi collaborate in a novel pathway for beta-catenin degradation linked to p53 responses. Mol Cell 2001;7:915-26.

7. Filipek A, Jastrzebska B, Nowotny M, Kwiatkowska K, Hetman M, Surmacz L, et al. $\mathrm{Ca}^{2+}$-dependent translocation of the calcyclin-binding protein in neurons and neuroblastoma NB-2a cells. J Biol Chem 2002;277:21103-9.

8. Wu J, Tan X, Peng X, Yuan J, Qiang B. Translocation and phosphorylation of calcyclin binding protein during retinoic acidinduced neuronal differentiation of neuroblastoma SH-SY5Y cells. J Biochem Mol Biol 2003;36:354-8.
9. Zhao Y, You H, Liu F, An H, Shi Y, Yu Q, et al. Differentially expressed gene profiles between multidrug resistant gastric adenocarcinoma cells and their parental cells. Cancer Lett 2002; 85:211-8.

10. D'Souza CD, Kadival GV, Samuel AM. Use of the recombinant 38-kDa antigen of Mycobacterium tuberculosis as an immunogen for specific antisera production. Microbiol Immunol 1994;38:797800.

11. Shi Y, Han Y, Wang X, Zhao Y, Ning X, Xiao B, et al. MGr1-Ag is associated with multidrug-resistant phenotype of gastric cancer cells. Gastric Cancer 2002;5:154-9.

12. Shi Y, Zhai H, Wang X, Wu H, Ning X, Han Y, et al. Multidrugresistance-associated protein MGr1-Ag is identical to the human 37-kDa laminin receptor precursor. Cell Mol Life Sci 2002;59: 1577-83.

13. Larsen AK, Escargueil AE, Skladanowski A. Resistance mechanisms associated with altered intracellular distribution of anticancer agents. Pharmacol Ther 2000;85:217-29.

14. Fan D, Liu X. New progresses in researches on multidrug resistance in gastric cancer. Chin J Digest 2000;20:77-8.

15. Ross DD. Novel mechanisms of drug resistance in leukemia. Leukemia 2000;14:467-73.

16. Litman T, Druley TE, Stein WD, Bates SE. From MDR to MXR: new understanding of multidrug resistance systems, their properties and clinical significance. Cell Mol Life Sci 2001;58:931-59.

17. Gurel S, Yerci O, Filiz G, Dolar E, Yilmazlar T, Nak SG, et al. High expression of multidrug resistance-1 (MDR-1) and its relationship with multiple prognostic factors in gastric carcinomas in patients in Turkey. J Int Med Res 1999;27:79-84.

18. Yeh KH, Chen CL, Shun CT, Lin JT, Lee WJ, Lee PH, et al. Relatively low expression of multidrug resistance-1 (MDR-1) and its possible clinical implication in gastric cancers. J Clin Gastroenterol 1998;26:274-8

19. Alexander D, Yamamoto T, Kato S, Kasai S. Histopathological assessment of multidrug resistance in gastric cancer: expression of P-glycoprotein, multidrug resistance-associated protein, and lungresistance protein. Surg Today 1999;29:401-6.

20. Takebayashi Y, Akiyama S, Natsugoe S, Hokita S, Niwa K, Kitazono M, et al. The expression of multidrug resistance protein in human gastrointestinal tract carcinomas. Cancer 1998;82:6616.

21. Fan KC, Fan DM, Cheng LF, Li CH. Expression of multidrug resistance related markers in gastric cancer. Anticancer Res 2000;20:4809-14.

22. Chung YM, Park S, Park JK, Kwon OY, Kim YK, Ro HK, et al. Establishment and characterization of 5-fluorouracil-resistant gastric cancer cells. Cancer Lett 2000;159:95-101.

23. Takechi T, Koizumi K, Tsujimoto H, Fukushima M. Screening of differentially expressed genes in 5-fluorouracil-resistant human gastrointestinal tumor cells. Jpn J Cancer Res 2001;92:696-703.

24. Sinha P, Hutter G, Kottgen E, Dietel M, Schadendorf D, Lage H. Increased expression of annexin I and thioredoxin detected by two-dimensional gel electrophoresis of drug resistant human stomach cancer cells. J Biochem Biophys Methods 1998;37:10516.

25. An HZ, Zhou SJ, Fan DM. Establishment and characteristics of an adriamycin resistant human gastric carcinoma cell line (in Chinese with English abstract). Xiandai Xiaohua Bing Ji Neijing Zazhi (Mod J Dig Dis Endosc) 1997;2:108-10.

26. Filipek A, Jastrzebska B, Nowotny M, Kuznicki J. CacyBP/SIP, a calcyclin and Siah-1-interacting protein, binds EF-hand proteins of the S100 family. J Biol Chem 2002;277:28848-52.

27. Shyu RY, Huang SL, Jiang SY. Retinoic acid increases expression of the calcium-binding protein S100P in human gastric cancer cells. J Biomed Sci 2003;10:313-9. 